\title{
Primary health care and universal health coverage: Achieving health for all
}

\author{
Zoi Tsimtsiou ${ }^{1,2 *}$ \\ ${ }^{1}$ Department of Hygiene, School of Medicine, Aristotle University of Thessaloniki, Greece \\ ${ }^{2}$ Urban Health Center of Evosmos, Thessaloniki, Greece
}

The movement towards universal health coverage (UHC) is now one of the most prominent global health policies [1]. According to the United Nations Sustainable Development Goals, all UN Member States have agreed to try to achieve Universal Health Coverage by 2030 [2]. This includes financial risk protection, access to quality essential health-care services and access to safe, effective, quality and affordable essential medicines and vaccines for all [2]. As more countries make commitments to universal health coverage, they face challenges on how to quantify it and track progress towards its key goals, both in terms of health services and financial protection coverage. The Joint WHO/World Bank Group report released on 2015 entitled 'Tracking universal health coverage: First global monitoring report" provided guidance about how states can achieve universal health coverage and build more resilient health systems [3].

Emphasis should be placed on the role of primary health care in achieving universal health coverage [4]. Health for all is an indispensable need which must be integrated into an overall framework for organising and delivering care based on a patientcentered, efficient, fair, and cost-effective way [5]. According to the World Health Report 2008: Primary Health Care (Now More Than Ever), primary health care can provide such a framework [6]. Patientcenteredness, comprehensiveness, integration and continuity of care are among primary health care's core features that have been repeatedly associated with better health outcomes, cost-effectiveness and higher user satisfaction $[7,8]$. However, it is recognised that primary care can offer much more than reduction of costs. A recent study on 102 low- and middle-income countries reported that broader coverage of primary care services was linked to longer life expectancy, lower infant mortality and lower under-five mortality, suggesting that investment in primary care is a wise choice [9]. The increased accessibility even to deprived populations, the provision of long term patient-centered care, the emphasis on prevention, the reduction of unnecessary medical care are among the benefits that a strong primary health system can offer, narrowing the gap between socially deprived and advantaged populations [5]. Strengthening primary health care systems, combined with financial protection by universal coverage, seems to be the gold pathway to achieve health for all.

\section{References}

1. Rumbold B, Baker R, Ferraz O, Hawkes S, Krubiner C, et al. (2017) Universal health coverage, priority setting, and the human right to health. Lancet 17: 30931-30935. [Crossref]

2. WHO (2016) Health topics, World Health Organization.

3. WHO (2016) Tracking universal health coverage: First global monitoring report Health statistics and information systems World Health Organization /World Bank Group report.

4. Rao M, Pilot E (2014) The missing link--the role of primary care in global health. Glob Health Action 7: 23693. [Crossref]

5. Stigler FL, Macinko J, Pettigrew LM, Kumar R, van Weel C (2016) No universal health coverage without primary health care. Lancet 387: 1811. [Crossref]

6. WHO (2008) World health report, The World Health Report 2008: Primary Health Care (Now More Than Ever).

7. O'Malley AS, Rich EC, Maccarone A, DesRoches CM (2015) Reid RJ. Disentangling the Linkage of Primary Care Features to Patient Outcomes: A Review of Current Literature, Data Sources, and Measurement Needs. J Gen Intern Med 30: 576-585.

8. Fan VS, Burman M, McDonell MB, Fihn SD (2005) Continuity of care and other determinants of patient satisfaction with primary care. J Gen Intern Med 20: 226-233. [Crossref]

9. Hsieh VC, Wu JC, Wu TN, Chiang TL (2015) Universal coverage for primary health care is a wise investment: evidence from 102 low- and middle-income countries.Asia Pac J Public Health 27: NP877-NP886.
Copyright: (C2017 Tsimtsiou Z. This is an open-access article distributed under the terms of the Creative Commons Attribution License, which permits unrestricted use, distribution, and reproduction in any medium, provided the original author and source are credited.
Correspondence to: Zoi Tsimtsiou, Department of Hygiene, School of Medicine, Aristotle University of Thessaloniki, Greece, Tel: +306977086626; E-mail: zoitsimtsiou@yahoo.gr

Received: May 12, 2017; Accepted: May 15, 2017; Published: May 17, 2017 